\title{
Uso de metodologias ativas na promoção do autocuidado e adesão terapêutica com usuários de um centro de atenção psicossocial
}

Use of active methodologies in promoting self-care and therapeutic adherence with users of a psychosocial care center

Uso de metodologías activas en la promoción del autocuidado y la adherencia terapéutica con los usuarios de un centro de atención psicossocial

Amanda Guimarães Cunha ORCID: https://orcid.org/0000-0002-0893-3281

Centro Universitário Fibra, Brasil

E-mail: amandaguic18@gmail.com

Ana Flávia Lima da Silva

ORCID: https://orcid.org/0000-0002-4662-5597 Universidade do Estado do Pará, Brasil E-mail: aflima754@gmail.com

Ana Paula de Souza Mendes

ORCID: https://orcid.org/0000-0002-1831-541X Universidade do Estado do Pará, Brasil

E-mail: napaula000@gmail.com

Andressa Karolinny Costa de Oliveira ORCID: https://orcid.org/0000-0002-9108-7839

Universidade da Amazônia, Brasil

E-mail: andressakarolinnyscosta@gmail.com

Beatriz Stephany Conceição Braga ORCID: https://orcid.org/0000-0002-2686-3301

Universidade do Estado do Pará, Brasil

E-mail: beatriz.s.braga10@gmail.com

Brenda Caroline Martins da Silva ORCID: https://orcid.org/0000-0002-3474-2921

Universidade Federal do Pará, Brasil

E-mail: carol.brenda1994@gmail.com

Danielle Cardoso Portilho

ORCID: https://orcid.org/0000-0001-5905-0609

Universidade Federal do Amapá, Brasil

E-mail: daniellecardosoportilho@gmail.com

Denize Cardoso Portilho

ORCID: https://orcid.org/0000-0003-1754-1498

Universidade do Estado do Pará, Brasil

E-mail: denizeportilho60@gmail.com

Jamille Luciana Monteiro Nascimento

ORCID: https://orcid.org/0000-0003-4279-2125

Universidade do Estado do Pará, Brasil

E-mail: jamillelucianasz@gmail.com

Jéssica Maria Lins da Silva

ORCID: https://orcid.org/0000-0003-3218-6447

Universidade do Estado do Pará, Brasil E-mail: jeeh.sylva@gmail.com

Jordan da Silva Soeiro

ORCID: https://orcid.org/0000-0001-9092-0887 Universidade do Estado do Pará, Brasil E-mail: jordansoeiro@gmail.com

Lucinéia Ferreira Ferreira

ORCID: https://orcid.org/0000-0001-8957-1536 Universidade do Estado do Pará, Brasil

E-mail: lucineiaferreira4509@gmail.com

Matheus Ataide Carvalho

ORCID: https://orcid.org/0000-0001-9567-6799 Universidade do Estado do Pará, Brasil E-mail: mac8596@hotmail.com 
Patricia da Silva Ferreira

ORCID: https://orcid.org/0000-0001-7184-695X Universidade da Amazônia, Brasil

E-mail: ferreirapatty240@gmail.com

Pedro Henrique Carrias de Carvalho ORCID: https://orcid.org/0000-0002-5227-9133 Centro Universitário Fibra, Brasil E-mail: henrique.pedro715@gmail.com

Roseli Reis da Silva

ORCID: https://orcid.org/0000-0001-7512-1307 Faculdade Pan Amazônica, Brasil E-mail: rosydonova@gmail.com

\begin{abstract}
Resumo
Os transtornos mentais possuem etiologia diversa, sendo caracterizados pelas manifestações clínicas que cada indivíduo irá expressar. Em âmbito mundial, eles têm grande impacto no cenário da saúde, estima-se que $12 \%$ da carga mundial de doenças está diretamente ligada a essas patologias. A partir do exposto, objetivou-se nesse estudo relatar uma vivência de acadêmicos de enfermagem ocorrida em um centro de atenção psicossocial na região metropolitana de Belém-PA, ocorrida através da implementação de ações lúdicas no local, que visaram o fomento à adesão e continuidade terapêutica. O presente estudo classifica-se como descritivo, com abordagem qualitativa, do tipo relato de experiência, no qual se busca compreender as relações desenvolvidas embasados nos diferentes contextos. Fundamentou-se na metodologia da problematização do Arco de Charles Maguerez. Como resultado demonstrou-se a indispensabilidade de se trabalhar com metodologias alternativas com os usuários, já que esse público, em sua maioria, não conta com o apoio familiar e em decorrência do abandono, acabam optando em viver nas rua, e por isso apresentam-se em situação de risco, bem como são mais suscetíveis a abandonarem o tratamento oferecido no CAPS AD. Diante disso, é de grande valia deixar explicitado o quanto ações de educação em saúde com interações menos formais com a utilização de metodologias ativas são importantes na contribuição do autocuidado dos usuários, tal como no aprendizado dos acadêmicos.
\end{abstract}

Palavras-chave: Serviços de saúde mental; Assistência integral à saúde; Enfermagem.

\begin{abstract}
Mental disorders have different etiology, being characterized by the clinical manifestations that each individual will express. Worldwide, they have a major impact on the health scenario, it is estimated that $12 \%$ of the global disease burden is directly linked to these pathologies. Based on the above, the objective of this study was to report an experience of nursing students that took place in a psychosocial care center for alcohol and other drugs (CAPS AD, abbreviation in portuguese) in the metropolitan region of Belém-PA, which occurred through the implementation of playful actions on the site, which aimed at promoting adherence and continuity therapy. The present study is classified as descriptive, with a qualitative approach, of the experience report type, in which it seeks to understand the relationships developed based on different contexts. It was based on the methodology of problematizing the Arch of Charles Maguerez. As a result, it was demonstrated the indispensability of working with alternative methodologies with users, since this public, for the most part, does not have family support and as a result of abandonment, they end up choosing to live on the streets, at risk, as well as being more susceptible to abandoning the treatment offered at CAPS AD. Therefore, it is of great value to make it clear how much health education actions with less formal interactions with the use of active methodologies are important in the contribution of users' self-care, as well as in the learning of academics.
\end{abstract}

Keywords: Mental health services; Comprehensive health care; Nursing.

\title{
Resumen
}

Los trastornos mentales tienen diferente etiología, caracterizándose por las manifestaciones clínicas que expresará cada individuo. A nivel mundial, tienen un gran impacto en el escenario de salud, se estima que el $12 \%$ de la carga de morbilidad global está directamente relacionada con estas patologías. Con base en lo anterior, el objetivo de este estudio fue reportar una experiencia de estudiantes de enfermería que tuvo lugar en un centro de atención psicosocial de la región metropolitana de Belém-PA, que ocurrió a través de la implementación de acciones lúdicas en el sitio, las cuales tuvieron como objetivo promover la adherencia y la continuidad. terapia. El presente estudio se clasifica como descriptivo, con enfoque cualitativo, del tipo relato de experiencia, en el que se busca comprender las relaciones que se desarrollan a partir de diferentes contextos. Se basó en la metodología de problematizar el Arco de Carlos Maguerez. Como resultado, se demostró la indispensabilidad de trabajar con metodologías alternativas con los usuarios, ya que este público, en su mayor parte, no cuenta con apoyo familiar y como consecuencia del abandono, terminan optando por vivir en la calle. en riesgo, además de ser más susceptibles a abandonar el tratamiento ofrecido en CAPS AD. Por tanto, es de gran valor dejar claro cuánto son importantes las acciones de educación en salud con interacciones menos formales con el uso de metodologías activas en el aporte del autocuidado de los usuarios, así como en el aprendizaje de los académicos. 
Palabras clave: Servicios de salud mental; Atención integral de salud; Enfermería.

\section{Introdução}

Os transtornos mentais possuem etiologia diversa, sendo caracterizados pelas manifestações clínicas que cada indivíduo irá expressar. Em âmbito mundial, eles têm grande impacto no cenário da saúde, estima-se que 12\% da carga mundial de doenças está diretamente ligada a essas patologias (Santos \& Siqueira, 2010). No Brasil, o atendimento a esse público é feito a partir do Sistema Único de Saúde (SUS), que subsidia e implementa os tratamentos que esses indivíduos demandam, através da promoção de uma assistência integral, gratuita e equânime (Carvalho,2013).

Dentre os serviços ofertados pelo sistema de saúde brasileiro no âmbito da saúde mental, destacam-se os centros de atenção psicossocial (CAPS), que caracterizam-se por oferecer serviços abertos e interdisciplinares para indivíduos com transtornos mentais, desde que estejam com o quadro estabilizado, servindo como um suporte imprescindível para evitar quadros de reinternação e abandono terapêutico (Nasi \& Schneider, 2011).

Assim, esses centros representam um grande marco no cuidado ao paciente com demandas mentais, uma vez que oferta diversas atividades complementares extramuro que descaracterizam e mitigam a visão hospitalocêntrica, enfatizando a importância do fomento à reinserção social desses indivíduos na comunidade (Salles \& Barros, 2013).

Para tanto, dentre as atividades desenvolvidas, destacam-se as de caráter lúdico, uma vez que se utilizam de mecanismos de fácil compreensão e manejo, incentivando a participação dos usuários e tornando-os sujeitos ativos, posto que para o seu desenvolvimento é fundamental que ocorram intervenções constantes do público-alvo, que passa a delinear as atividades com base em suas demandas (Coscrato, Pina \& Mello, 2010).

Neste interim, enfatiza-se a figura do enfermeiro como profissional com papel educador dentro da assistência, que deve ser responsável por manter um vínculo de confiança com o paciente ao passo que o incentiva a tomar decisões conscientes sobre a sua saúde, promovendo o autocuidado e a manutenção de hábitos benéficos, em especial quando ligados à adesão terapêutica necessária para a implementação de um cuidado efetivo (Backes, Lino, Prado, Reibnitz \& Canaver, 2008).

A partir do exposto, objetivou-se nesse estudo relatar uma vivência de acadêmicos de enfermagem ocorrida em um centro de atenção psicossocial na região metropolitana de Belém-PA, ocorrida através da implementação de ações lúdicas no local, que visaram o fomento à adesão e continuidade terapêutica, fortalecimento de vínculos entre a equipe, familiares e usuários, bem como a promoção de estímulos ao autocuidado e a manutenção da qualidade de vida e bem-estar.

\section{Metodologia}

O presente estudo classifica-se como descritivo, com abordagem qualitativa, do tipo relato de experiência, no qual se busca compreender as relações desenvolvidas embasados nos diferentes contextos (Pereira, Shitsuka, Parreira, \& Shitsuka, 2018). Fundamentou-se na metodologia da problematização do Arco de Charles Maguerez, que parte do princípio da observação da realidade e definição de um problema para a definição das intervenções necessárias, associados em 5 fases: (1) Observação da realidade; (2) Levantamento de Pontos-chave; (3) Teorização; (4) Hipóteses de solução; e, (5) Retorno com aplicação à realidade (Berbel, 2011).

Por conseguinte, a observação da realidade partiu da vivência de acadêmicos de enfermagem durante o estágio obrigatório do componente curricular "Saúde Mental II", durante as consultas clínicas à usuários de um Centro de Atenção Psicossocial Álcool e outras Drogas (CAPS AD), localizado na região metropolitana de Belém. No referido local, foi identificado uma significativa demanda de usuários, de ambos os sexos, com dificuldades em externar aflições e situações que acabavam levando os mesmos ao uso de bebidas alcoólicas e/ou substâncias psicoativas, além do abandono a terapêutica realizada. 
Perante a situação descrita, os acadêmicos juntamente com o auxílio dos enfermeiros do CAPS AD levantaram os pontos-chave da problemática, chegando a conformidade da necessidade de uma ação educativa em saúde, abrindo um espaço fora do consultório e aproveitando as atividades referente ao Setembro Amarelo, mês alusivo à prevenção ao suicídio, já que na cidade rotineiramente noticiavam-se casos de suicídio de pessoas suscetíveis, como os usuários em questão.

A teorização ocorreu através das buscas em bases digitais de estudos científicos para auxiliar o desenvolvimento teórico e conduzir uma ação. Dessa forma, foram utilizadas as bases de dados: Scientific Eletronic Library Online (SCIELO), PUBMED e a Biblioteca Virtual em Saúde (BVS) (BIREME), das quais emergiram 21 estudos científicos, que serviram para embasar cientificamente a proposta de educação em saúde a ser realizada.

Assim sendo, dentro das hipóteses de solução, decidiu-se elaborar uma ação educativa direcionada aos usuários que frequentavam o CAPS AD. A ação foi dividida em dois momentos da seguinte forma: primeiramente, no dia da ação, os usuários presentes foram convidados a participar de uma palestra com coffee break, onde ocorreu uma breve explanação sobre a história do Setembro amarelo bem como sobre o suicídio propriamente dito; posteriormente, os mesmos participaram de uma dinâmica, a qual produziram desenhos e/ou diferentes tipos de demonstração abstrata, e por fim, os usuários externaram o significado das suas respectivas produções artísticas relacionando a própria vida e a temática da ação.

O convite à participação da palestra se deu por meio de uma breve conversa no dia anterior com os profissionais do CAPS AD para que houvesse um horário oportuno para que a maioria dos usuários presentes pudessem participar. Assim, a ação contou com exposição oral e o auxílio de recursos midiáticos, com slides, e posteriormente foram entregues lápis de cor, giz de cera e folhas de papel A4, sendo estes os mecanismos consolidadores da atividade proposta.

A aplicação na realidade ocorreu através do desenvolvimento da educação em saúde com o uso de tecnologias leves e exposição oral, sendo desenvolvida em um dia com abordagem teórica e prática. Ocorreu em um espaço fechado cedido pelo próprio CAPS, durando a manhã toda. No momento da ação fizeram-se presentes 15 usuários e três acompanhantes, além de uma enfermeira do local, que estava auxiliando na programação, e dos acadêmicos mediadores da ação.

\section{Resultados}

Como principais resultados obtidos a partir da aplicação do Arco de Maguerez, demonstrou-se a indispensabilidade de se trabalhar com metodologias alternativas com os usuários, já que esse público, em sua maioria, não conta com o apoio familiar e em decorrência do abandono, acabam optando em viver nas rua, e por isso apresentam-se em situação de risco, bem como são mais suscetíveis a abandonarem o tratamento oferecido no CAPS AD. Em vista disso, a palestra e a dinâmica pontuaram a necessidade do autocuidado, mental e físico, através do repasse de informações fundamentadas para retificar e correlacionar a saúde mental em detrimento a possíveis inclinações suicidas.

No dia da ação, durante a palestra, observou-se que alguns dos participantes encontravam-se tímidos e desconfortáveis, dois deles quiseram se retirar do local, mas como estavam acompanhados por seus respectivos familiares, foram convencidos a permanecer e participar, entretanto no desenrolar do processo da conversa e interação com os acadêmicos de enfermagem, percebeu-se que eles ficaram mais atentos e participativos, de maneira que a comunicação entre ambas as partes mostrou-se mais fluente e efetiva no decorrer da atividade.

Dessa forma, como implicações positivas da ação, pode-se destacar que o grupo compreendeu e foi sensibilizado quanto as orientações e as condutas que devem tomar acerca de sinais depressivos ou comportamentos suicidas, assim como acerca do consumo de bebidas alcoólicas e de substâncias psicoativas. Logo, partindo desse ponto da ação, foi possível tornar os usuários aptos a reconhecer sinais específicos em seu cotidiano que necessitem de um suporte imediato, além da importância de uma adesão contínua ao tratamento para a manutenção da qualidade de vida.

Outrossim, no que se refere a dinâmica da ação seguinte, verificou-se que durante as representações realizadas, os 
usuários utilizaram-se de desenhos e de frases como ferramenta para construir um ambiente seguro para a externalização de situações vivenciadas com o tratamento. Nesse momento foi evidente a complexidade pela qual cada indivíduo era submetido, por conta de fatores internos e externos, assim como também foi possível corroborar a influência dos profissionais no auxílio ao tratamento e a sensibilização desses indivíduos.

Em síntese, durante a exposição das produções, houve grande comoção após os relatos explicativos acerca do que estava sendo apresentado, isto se deve ao fato que quase todos possuíam pelo menos um relato pessoal ou familiar relacionado a tentativa do suicídio ou ao abandono do tratamento, ainda em sua maioria correlacionados ao uso de bebidas alcoólicas, o que resultou em ratificações sobre a necessidade e importância de um acompanhamento multidisciplinar para a melhora na qualidade de vida e no autocuidado, assim como promoção do bem-estar.

\section{Discussão}

Com base nos resultados apresentados neste estudo, evidenciou-se que o uso de metodologias ativas, em especial daquelas que utilizam a ludicidade, são de grande valor para a troca de conhecimentos e experiências entre os participantes, uma vez que propicia maior interação interpessoal ao passo que estabelece propriedades terapêuticas na consolidação da assistência prestada (Macedo et al., 2018).

Destarte, segundo Mineiro e D’Ávila (2019), a ludicidade configura-se como um mecanismo de ensino-aprendizagem inestimável, uma vez que o usuário pode através deste método compreender a necessidade da manutenção de hábitos benéficos e ter sua autonomia incentivada para o estabelecimento de um autocuidado diligente e eficaz de acordo com as suas demandas específicas.

Ademais, reitera-se a importância da promoção da interação entre os usuários e os profissionais do local, fortalecendo vínculos e mitigando barreiras comunicativas, propiciando, assim, uma assistência mais empática e integral. Em consonância com o exposto, Guerrero, Mello, Andrade e Erdmann (2013) dispõem sobre a importância da confiança e da construção de uma relação segura e saudável com o paciente para a implementação conjunta de intervenções que fomentem a assistência integral e humanizada na rede de cuidados.

Para mais, a escuta e conversas ocorridas durante a ação mostraram-se efetivas, uma vez que permitiram a identificação de necessidades específicas e lacunas assistenciais acerca da terapêutica, bem como possibilitou conhecer os seus reflexos em cada usuário, momento no qual ficaram evidentes as dificuldades e as intervenções a serem implantadas nos hábitos de saúde destes indivíduos. Assim, destaca-se que uma atenção qualificada é fundamental para a promoção de um cuidado centrado no usuário e em suas carências (Maynart, Albuquerque, Brêda, \& Jorge, 2014).

Além disso, também enfatiza-se a relevância da participação de acadêmicos nessas atividades, visto que a partir disso eles podem adquirir aptidões e desenvolver habilidades e competências que os auxiliarão em sua gestão de cuidados futuramente. Para mais, essa participação também influencia diretamente na construção de um olhar holístico e equânime do profissional, a partir do conhecimento de diferentes demandas e da aplicação de intervenções diretamente na comunidade, aumentando o contato com a realidade dos usuários dos serviços (Silva et al., 2020).

Portanto, salienta-se a partir deste relato que as atividades desenvolvidas contribuíram significativamente para a promoção da saúde e bem-estar dos usuários, assim como para o fomento ao autocuidado e a manutenção do tratamento. Dessa forma, ratifica-se a necessidade de uma construção contínua e consolidada de uma assistência biopsicossocial, mitigando condutas rasas e tecnicistas e atendendo as demandas dos pacientes de maneira holística, propiciando, assim, um cuidado integral e humanizado a esses indivíduos (Perboni, Silva \& Oliveira, 2019).

As limitações encontradas neste artigo relacionam-se diretamente ao baixo quantitativo de usuários participantes, tendo em vista o grande número de frequentadores do CAPS AD, o que acaba interferindo na variabilidade de resultados 
apresentados.

\section{Considerações Finais}

$\mathrm{Na}$ atual conjuntura, o modelo de ensino tradicional vem sendo gradativamente substituído por novas tendências e implicações as quais apontam para a necessidade da formação de um profissional crítico-reflexivo, capaz de transverter a realidade social do seu cotidiano em benefícios acessíveis para a comunidade ao qual é inserido, tendo em vista proveitos coletivos que influenciarão diretamente na qualidade assistencial futura.

Dessa forma, a partir dos resultados obtidos neste estudo, evidenciou-se que a assistência prestada a esse público deve corresponder a todas as demandas apresentadas, não focando apenas no viés tecnicista, e sim enxergando o indivíduo de maneira equânime e integral, para que assim, situações como o abandono do tratamento e riscos secundários, como o de suicídio apontado por alguns usuários deste estudo, sejam continuamente mitigados pela equipe multiprofissional.

Diante disso, espera-se que este trabalho possa fomentar a criação de estratégias para a melhora na qualidade de vida do público em questão. Ademais, é de grande valia explicitar o quanto ações de educação em saúde com interações menos formais e com a utilização de metodologias ativas são importantes na sensibilização dos usuários. Outrossim, ratifica-se que a presença de acadêmicos atuando e intervindo diretamente nas diferentes realidades assistenciais é fundamental para o desenvolvimento de aptidões necessárias à sua assistência. Para mais, sugere-se para estudos futuros um maior número de participantes e a aplicação de atividades em diferentes centros de atenção psicossocial, visando a disseminação de ações que promovam a saúde deste público, bem como uma maior variedade de resultados.

\section{Referências}

Backes, V. M. S., Lino, M. M., Prado, M. L., Reibnitz, K. S., \& Canaver, B. P. (2008). Competência dos enfermeiros na atuação como educador em saúde. Revista Brasileira de Enfermagem, 61(6), 858-865.

Berbel, N. A. N. (2011). As metodologias ativas e a promoção da autonomia de estudantes. Semina: Ciências Sociais e Humanas, 32(1), 25-40.

Carvalho, G. (2013). A saúde pública no Brasil. Estudos Avançados, 27(78), 7-26.

Coscrato, G., Pina, J. C., \& Mello, D. F. (2010). Utilização de atividades lúdicas na educação em saúde: uma revisão integrativa da literatura. Acta Paulista de Enfermagem, 23(2), 257-263.

Falkenberg, M. B., Mendes, T. d. P. L., Moraes, E. P. d., \& Souza, E. M. d. (2014). Educação em saúde e educação na saúde: conceitos e implicações para a saúde coletiva. Ciência e saúde coletiva, 19(3), 847-52.

Guerrero, P., Mello, A. L. S. F., Andrade, S. R., \& Erdmann, A. L. (2013). O acolhimento como boa prática na atenção básica à saúde. Texto \& Contexto Enfermagem, 22(1), 132-140.

Macedo, K. D. S., Acosta, B. S., Silva, E. B., Souza, N. S., Beck, C. L. C., \& Silva, K. K. D. (2018). Metodologias ativas de aprendizagem: caminhos possíveis para inovação no ensino em saúde. Escola Anna Nery, 22(3), e20170435.

Maynart, W. H. C., Albuquerque, M. C. S., Brêda, M. Z., \& Jorge, J. S. (2014). A escuta qualificada e o acolhimento na atenção psicossocial. Acta Paulista de Enfermagem, 27(4), 300-304.

Mineiro, M., \& D’Ávila, C. (2019). Ludicidade: compreensões conceituais de pós-graduandos em educação. Educação e Pesquisa, 45 , e208494.

Nasi, C., \& Schneider, J. F. (2011). O Centro de Atenção Psicossocial no cotidiano dos seus usuários. Revista da Escola de Enfermagem da USP, 45(5), 11571163 .

Perboni, J. S., Silva, R. C., \& Oliveira, S. G. (2019). A humanização do cuidado na emergência na perspectiva de enfermeiros: enfoque no paciente politraumatizado. Interações (Campo Grande), 20(3), 959-972.

Prado, M. L. d., Velho, M. B., Espíndola, D. S., Sobrinho, S. H., \& Backes, V. M. S. (2012). Arco de Charles Maguerez: refletindo estratégias de metodologia ativa na formação de profissionais de saúde. Escola Anna Nery, 16(1), 172-177.

Salles, M. M., \& Barros, S. (2013). Exclusão/inclusão social de usuários de um centro de atenção psicossocial na vida cotidiana. Texto \& Contexto Enfermagem, 22(3), 704-712.

Santos, E. G., \& Siqueira, M. M. (2010). Prevalência dos transtornos mentais na população adulta brasileira: uma revisão sistemática de 1997 a 2009. Jornal Brasileiro de Psiquiatria, 59(3), 238-246. 
Research, Society and Development, v. 10, n. 1, e54910111853, 2021

(CC BY 4.0) | ISSN 2525-3409 | DOI: http://dx.doi.org/10.33448/rsd-v10i1.11853

Silva, J. M. L., Monteiro, A. J. C., Coutinho, E. S., Cruz, L. B. S., Araújo, L. T., Dias, W. B, \& Costa, P. V. D. P. (2020). O brinquedo terapêutico instrucional como ferramenta na assistência oncológica infantil. Research, Society and Development, 9 (7), 1-14. 